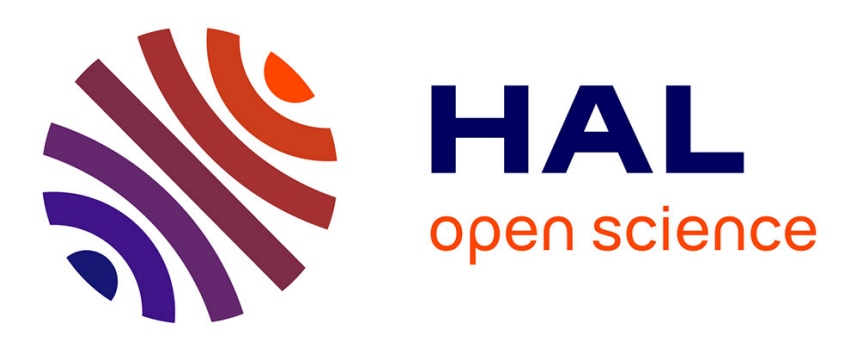

\title{
Optimal campaign strategy for social media marketing with a contrarian population
}

Vineeth Varma, Bikash Adhikari, Irinel-Constantin Morârescu, Elena Panteley

\section{To cite this version:}

Vineeth Varma, Bikash Adhikari, Irinel-Constantin Morârescu, Elena Panteley. Optimal campaign strategy for social media marketing with a contrarian population. 10th International Conference on Network Games, Cuntrol and Optimization, NETGCOOP 2021, Sep 2021, Cargèse, France. hal03365824

\section{HAL Id: hal-03365824 \\ https://hal.science/hal-03365824}

Submitted on 12 Oct 2021

HAL is a multi-disciplinary open access archive for the deposit and dissemination of scientific research documents, whether they are published or not. The documents may come from teaching and research institutions in France or abroad, or from public or private research centers.
L'archive ouverte pluridisciplinaire HAL, est destinée au dépôt et à la diffusion de documents scientifiques de niveau recherche, publiés ou non, émanant des établissements d'enseignement et de recherche français ou étrangers, des laboratoires publics ou privés. 


\title{
Optimal campaign strategy for social media marketing with a contrarian population *
}

\author{
Vineeth S. Varma ${ }^{1[0000-0001-8762-2790]}$, Bikash Adhikari ${ }^{1}$, Irinel-Constantin \\ Morărescu ${ }^{1}$, and Elena Panteley ${ }^{2}$ \\ 1 Université de Lorraine, CRAN, UMR 7039 and the CNRS, CRAN, UMR 7039, \\ France, vineeth.satheeskumar-varma@univ-lorraine.fr. \\ ${ }^{2}$ Laboratoire des signaux et systèmes, CNRS, 3 rue Joliot Curie, 91192 \\ Gif-sur-Yvette, France
}

\begin{abstract}
We address formally the problem of opinion dynamics when the social network composed of conformists and contrarians are not only influenced by their neighbors, but also by an external influential entity referred to as a marketer. The population of contrarians tries to have an opinion that is the opposite of the opinion held by the conformists. The influential entity tries to sway the overall opinion as close as possible to the desired opinion by using a specific influence budget. The main technical issue addressed is finding how the marketer should allocate its budget among the agents such that the agents' opinion will be as close as possible to the desired opinion while taking into account the behavior of the contrarian population. Our main results show that the marketer has to prioritize certain agents over others based on their initial condition, their influence power in the social graph and the population class they belong to. Numerical examples illustrate the analysis.
\end{abstract}

\section{Introduction}

During the last decade, social networks gained increasing importance in our daily life. Consequently, more and more companies are using digital social networks to promote specific goods and/or ideas. This motivated the scientific community to give more attention to the analysis of opinion dynamics in social networks. This is a challenging task since human behavior is very different from one individual to another and the interactions in the network can change over time. Various mathematical models $[7,9,6,10,1,4]$ have been proposed in order to capture different features of this complex dynamics. Empirical models based on in vitro and in vivo experiments have also been developed $[5,16,13]$.

Some mathematical model target consensus as collective asymptotic behavior of the network $[7,9]$ while some others lead to the network clustering $[10,1,14]$. In order to enforce consensus some recent studies propose the control of one or few agents (see $[3,8]$ ). Besides these methods of controlling opinion dynamics towards consensus, we also find few attempts to control the discrete-time dynamics of

\footnotetext{
* Supported by CEFIPRA under the project 6001-1/2019
} 
opinions such that, as many as possible reach a certain set after a finite number of influences [11]. In very recent work ([15]) the authors developed a formal method for the optimal space-time allocation of a budget allowing an influencer to bring the consensus value of the network as close as possible to a desired value.

It is noteworthy that most of the proposed mathematical models consider that the opinions of two interacting individuals are approaching one to another. Although this behavior seems to be sociologically accepted, one can also provide real-life situations in which interactions are antagonistic. In this case, the distance between the opinions of two interacting individuals will increase (see $[1,12$, 17]). To the best of our knowledge, there exists no attempt to control this kind of opinion dynamics, and only analysis of the asymptotic behavior is reported.

In this paper, we consider the challenging problem that requires to minimize the distance between the average of opinions and a desired value using a given control/marketing budget over a social network split into two groups. Basically, this social network with the contrarian population represents a model for real cases such as supporters of competing teams, parties, etc. On top of this assumption on the network structure, we also assume that the maximal marketing influence cannot instantaneously make the opinion of one individual to be equal with the desired value.

To provide a mathematical model we consider that the opinion dynamics is fast enough such that we can assume it evolves in continuous time and we want to design a marketing strategy that minimizes the distance between the average of the opinions and the desired value after a campaign with certain budget constraints. This results in a linear-impulsive closed-loop dynamics in which the jumps are controlled by the influencer. Our main result shows that the optimal control strategy consists of influencing as much as possible the most central/popular (see [2] for a formal definition of centrality) individuals of the network.

It is worth highlighting that in this study we do not control the state of the influencing entity which is assumed to be constant. Instead, we control the influence weight that the marketer can have on different individuals of the social network. It was shown in [15] that this approach allows highlighting the effectiveness of targeted marketing with respect to broadcasting strategies when budget constraints are present.

Notation. Let $\mathbb{R}:=(-\infty, \infty), \mathbb{R}_{\geq 0}:=[0, \infty)$, and $\mathbb{Z}_{\geq 0}:=\{0,1,2, \ldots\}$. We use $E$ for the expectation taken over the relevant stochastic variables and $\mathbf{1}(\cdot)$ for the indicator function, taking the value 1 when the condition is satisfied and 0 otherwise.

\section{Problem statement}

We consider a social network populated by agents belonging to the set $\mathcal{V}:=$ $\{1,2, \ldots, N\}$ with the connections given by $a_{i, j}$ indicating the influence of agent $j$ on agent $i$. All agents belong to the class of conformists denoted by the set 
$\mathcal{V}^{+}$or to the class of contrarians denoted by $\mathcal{V}^{-}$. We use $c_{n} \in\{1,-1\}$ to denote the agent class with $c_{n}=1$ for all $n \in \mathcal{V}^{+}$and $c_{n}=-1$ for all $n \in \mathcal{V}^{-}$. These two sets are non-intersecting and may be interpreted as two hostile camps as considered in [17].

The opinion of all agents belongs to the interval $[-1,1]$ and we denote the collection of all opinions by $x \in[-1,1]^{N}$. The internal opinion dynamics of the network is given by

$$
\dot{x}_{i}=\sum_{j \in \mathcal{V}} a_{i, j} x_{j}-\left|a_{i, j}\right| x_{i}
$$

for all $i \in \mathcal{V}$. The above dynamics is similar to a standard consensus model as in [6] but $a_{i, j}$ may be positive or negative depending on the interaction type.

In this work, we focus on antagonistic interaction between agents that belong to different classes and standard consensus interactions among agents of the same class, i.e., contrarians or conformist. Basically, this implies that $a_{i, j}=c_{i} c_{j}\left|a_{i, j}\right|$. As a result of this extra term, agents $i$ and $j$ will have a consensus only if $i, j$ belong to the same class. If they belong to different classes, agent $i$ will try to have an opinion in opposition to agent $j$. We can write the overall dynamics for all agents as

$$
\dot{x}=-L x
$$

where $L$ is a Laplacian-like matrix with

$$
L_{i, j}= \begin{cases}\sum_{k \in \mathcal{V}}\left|a_{i, k}\right| & \text { for all } i=j \in \mathcal{V}, \\ -a_{i, j} & \text { for all } i \neq j \in \mathcal{V}\end{cases}
$$

The influence of the external entity (marketer) with a desired opinion $d$ is limited to a campaign occurring at $t=0$. We model this influence through an impulsive control/jump at $t=0$. Due to the contrarian population, the marketer may also desire to bring some agents closer to the opinion $-d$ but has must select the best agents due to budget constraints. We, therefore, model the impulsive control as follows

$$
x_{n}\left(0^{+}\right)=\left(1-\left|u_{n}\right|\right) x_{n}(0)+u_{n} d
$$

for all $n \in \mathcal{V}$ with $u_{n} \in[-\bar{u}, \bar{u}]$, with $\bar{u}$ denoting the control saturation and $\sum_{n \in \mathcal{V}}\left|u_{n}\right| \leq B$ is the budget constraint.

The main objective of this paper is to provide an optimal control $u$ such that the distance between the average opinion of all agents and the desired opinion $d \in\{-1,1\}$, given by $\left|\frac{1_{N}^{\top} x}{N}-d\right|$ is minimized asymptotically. Given $x_{0} \in[-1,1]^{N}$, the optimization problem can be stated formally as follows

$$
\begin{gathered}
\text { Minimize }_{u} J(u):=\lim _{t \rightarrow \infty}\left|\frac{1_{N}^{\top} x(t)}{N}-d\right| \\
\text { where } \dot{x}(t)=-L x(t), x(0)=x_{0} \forall t>0, \\
\text { under (4) with } u_{n} \in[-\bar{u}, \bar{u}] \forall n \in \mathcal{V} \\
\text { and } \sum_{n \in \mathcal{V}}\left|u_{n}\right| \leq B .
\end{gathered}
$$




\section{Analysis}

To begin our analysis, we first characterize the asymptotic opinions of the agents under the dynamics (2). For this purpose, we rely on results established in [17].

Proposition 1. Given the opinions $x\left(0^{+}\right)$formed after the campaign, we have

$$
\lim _{t \rightarrow \infty} x(t)=c v^{\top} x\left(0^{+}\right)
$$

where $v$ is the left eigen-vector of the matrix $L$ associated with eigenvalue 0 with $\sum_{n \in \mathcal{V}}\left|v_{n}\right|=1$ and $\operatorname{sign}\left(v_{n}\right)=\operatorname{sign}\left(c_{n}\right)$.

Proof. First, we use Lemma 1 in [17] to conclude that if (2) establishes modular consensus (consensus in absolute value of each $x_{n}$, then

$$
\lim _{t \rightarrow \infty} x(t)=\rho v^{\top} x\left(0^{+}\right)
$$

where $\rho_{n} \in\{1,-1\}$ for all $n \in \mathcal{V}$. By construction in (3), we have that $a_{i, j}$ is positive for $i \neq j$ only when they both belong to the same set $\mathcal{V}^{+}$or $\mathcal{V}^{-}$and is negative otherwise. This implies that $\mathcal{V}^{+}$or $\mathcal{V}^{-}$form hostile camps as defined in [17], allowing us to apply Lemma 2 from [17]. This states that modular consensus is established for quasi-strongly connected graphs with $\rho_{i}=-\rho_{j}$ for all $i, j$ in opposite camps. Since we pick $\operatorname{sign}\left(v_{n}\right)=\operatorname{sign}\left(c_{n}\right)$, we have that $\rho_{n}=c_{n}$.

Proposition 1 allows us to study the impact of the control on the cost function in 5 . We provide the optimal control $u$ which will minimize this cost in the following. We define a sorting index

$$
\gamma_{n}:=\left|v_{n}\right|\left|c_{n} d-x_{n}(0)\right|
$$

Theorem 1. If $\operatorname{card}\left(\mathcal{V}^{+}\right)=\operatorname{card}\left(\mathcal{V}^{-}\right)$, the final cost is invariant to the control and $J(u)=1$ for all $u$. If $\operatorname{card}\left(\mathcal{V}^{+}\right)>\operatorname{card}\left(\mathcal{V}^{-}\right)$and the desired opinion be $d$, then the optimal control $u^{*}$ is given by

$$
u_{\mathrm{o}(n)}^{*}=\left\{\begin{array}{lll}
c_{\mathrm{o}(n)} \bar{u} & \text { if } & n \leq\left\lfloor\frac{B}{\bar{u}}\right\rfloor \\
B c_{\mathrm{o}(n)}-c_{\mathrm{o}(n)} \bar{u}\left\lfloor\frac{B}{\bar{u}}\right\rfloor & \text { if } & n=\left\lfloor\frac{B}{\bar{u}}\right\rfloor+1 \\
0 & \text { otherwise } &
\end{array}\right.
$$

where o : $\mathcal{V} \rightarrow \mathcal{V}$ is a bijection such that $\gamma_{\mathrm{o}(1)} \geq \gamma_{\mathrm{o}(2)} \geq \cdots \geq \gamma_{\mathrm{o}(N)}$. When $\operatorname{card}\left(\mathcal{V}^{+}\right)<\operatorname{card}\left(\mathcal{V}^{-}\right)$, setting $-d$ as the desired opinion and applying $u^{*}$ minimizes the cost $J(u)$.

Proof: First, we rewrite the minimization (5) problem as follows

$$
\begin{gathered}
\text { Minimize }_{u} \lim _{t \rightarrow \infty}\left|\frac{1_{N}^{\top} x(t)}{N}-d\right| \\
\text { where } \dot{x}(t)=-L x(t), \forall t>0, \\
x_{n}\left(0^{+}\right)=\left(1-u_{n}^{+}-u_{n}^{-}\right) x_{n}(0)+u_{n}^{+} d-u_{n}^{-} d, \forall n \in \mathcal{V} \\
u_{n}^{+}, u_{n}^{-} \in[0, \bar{u}], \text { such that } u_{n}^{+} u_{n}^{-}=0, \quad \forall n \in \mathcal{V}, \\
\sum_{n \in \mathcal{V}} u_{n}^{+}+u_{n}^{-} \leq B .
\end{gathered}
$$


in order to separate the positive and negative control action. Note that we have that

$$
\lim _{t \rightarrow \infty} x(t)=c v^{\top} x\left(0^{+}\right)
$$

since the dynamics in $(0, \infty)$ is given by $\dot{x}=-L x$ and using [1].

Denoting $x^{*}:=v^{\top} x\left(0^{+}\right)$, which is a scalar and belongs to the interval $[-1,1]$, we have that the final cost is given by

$$
J=\left|\frac{\sum_{n \in \mathcal{V}} c_{n}}{N} x^{*}-d\right|=\left|\frac{\operatorname{card}\left(\mathcal{V}^{+}\right)-\operatorname{card}\left(\mathcal{V}^{-}\right)}{N} x^{*}-d\right| .
$$

We use $G:=\left|\frac{\operatorname{card}\left(\mathcal{V}^{+}\right)-\operatorname{card}\left(\mathcal{V}^{-}\right)}{N}\right|$. Therefore,

1. if $\operatorname{card}\left(\mathcal{V}^{+}\right)=\operatorname{card}\left(\mathcal{V}^{-}\right)$, we have $J=d$ for any $x^{*}$,

2. if $\operatorname{card}\left(\mathcal{V}^{+}\right)>\operatorname{card}\left(\mathcal{V}^{-}\right)$, we have $J=\left|G x^{*}-d\right|$,

3. and if $\operatorname{card}\left(\mathcal{V}^{+}\right)<\operatorname{card}\left(\mathcal{V}^{-}\right), J=\left|G x^{*}+d\right|$.

This implies that minimizing the final cost $J$ is equivalent of minimizing $\left(x^{*}-d\right)^{2}$ for case 2 and $\left(x^{*}+d\right)^{2}$ for case 3 . We have

$$
\begin{array}{ll}
\frac{\partial\left(\left(d-x^{*}\right)^{2}\right)}{\partial u_{n}^{+}}=-2\left(d-x^{*}\right) v_{n}, & \frac{\partial\left(\left(d-x^{*}\right)^{2}\right)}{\partial u_{n}^{-}}=2\left(d-x^{*}\right) v_{n} \\
\frac{\partial\left(\left(d+x^{*}\right)^{2}\right)}{\partial u_{n}^{+}}=2\left(d+x^{*}\right) v_{n}, & \frac{\partial\left(\left(d+x^{*}\right)^{2}\right)}{\partial u_{n}^{-}}=-2\left(d+x^{*}\right) v_{n} .
\end{array}
$$

In the two cases cases, $2\left(d-x^{*}\right)$ and $2\left(d+x^{*}\right)$ are respectively positive functions which hold true for all $n$. Applying Lemma 1 (provided in the Appendix) for each case, we have the result by setting $u_{n}=u_{n}^{+}-u_{n}^{-}$.

Theorem 1 provides the control strategy to be implemented in order to minimize the cost $J(u)$, which implies minimizing the distance between the average final opinion and the desired opinion. When the number of contrarians and conformists are the same, the opinions are polarised around 0 in a symmetric fashion. Therefore, the final average opinion is always 0 , leading to a fixed cost of $J(u)=|d|$.

Nominally, we assume that the set of contrarians is a minority, i.e., $\operatorname{card}\left(\mathcal{V}^{+}\right)>$ $\operatorname{card}\left(\mathcal{V}^{-}\right)$. In this case, we select the most influential agents based on the index $\gamma$ which depends on the vector centrality $v$ as well as the distance to the desired opinion. However, since the contrarians oppose the conformists, the optimal strategy is to push the contrarians closer to $-d$ and the conformists closer to $d$. Since the conformists are a majority, the minimal cost is when all conformists are at $d$ and all contrarians at $-d$. The control in Theorem 1 precisely achieves this, i.e. it brings the conformists as close as possible to $d$ and the contrarians as close as possible to $-d$, under the budget constraints.

Note that when $\operatorname{card}\left(\mathcal{V}^{-}\right)=0$, i.e. there are no antagonistic relations, the optimal control $u^{*}$ in Theorem 1 matches the result in [15] (which considers $a_{i, j} \geq 0$ for all $i, j$ ) as expected. When the population of contrarians are larger, i.e. $\operatorname{card}\left(\mathcal{V}^{+}\right)<\operatorname{card}\left(\mathcal{V}^{-}\right)$, the minimal cost occurs when all conformists are at $-d$ and all contrarians at $d$. This is achieved by simply setting the new desired opinion to be $-d$ and applying the control in Theorem 1. 


\section{Numerical illustration}

To demonstrate and compare the numerical results the following budget allocation strategies are adopted:

Strategy 1: Optimal Budget Allocation, where the budget is allocated to agents according to Theorem 1.

Strategy 2: Uniform Budget Allocation, where budget is allocated uniformly to all the agents with negative control for contrarians, i.e. $u_{i}=c_{i} \frac{B}{N}$.

Strategy 3: Positive Budget Allocation, where budget is allocated uniformly and an identical control is applied to all the agents, i.e. $u_{i}=\frac{B}{N}$.

Strategy 3 corresponds to traditional advertising campaigns through Television or radio broadcasts as the same advertising action is applied to all agents regardless of their individual characteristics. The network structure we consider is a strongly connected directed graph with 10 nodes $(\mathrm{N}=10)$ and the number of conformists is greater than that of the contrarians $\left(|V|^{+}>|V|^{-}\right)$. The strongly connected graph is represented in Figure 1. The initial opinions are uniformly chosen between $[-1,1]$ and represented in Table 1 .

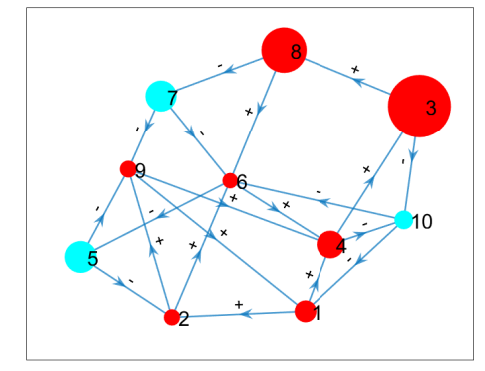

Fig. 1: Red and cyan nodes represent conformists and contrarians, respectively with the size scaled based on vector centrality $v$.

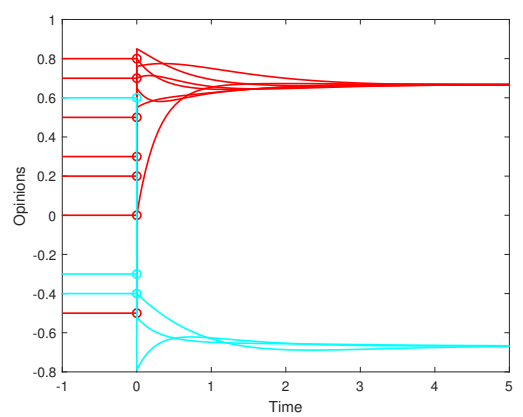

Fig. 2: Opinion dynamics with the control applied impulsively at $t=0$, red and cyan plots represent conformists and contrarians.

In Figure 1, the red and cyan nodes represent the conformist and the contrarian agents, respectively and the size of the nodes represents the agent's centrality. The centralities of all the agents are also represented in Table 1. As stated in Section 2, positive and negative values of $c$ represents conformists and contrarians respectively. Consider that the budget $B=4$, the maximum control that can be allocated to each agent $\bar{u}=0.7$, and opinions before the campaign as given in Table 1. When no control action is applied, we see that $J(0)=0.8877$. Using strategy 1, budget allocation is performed based on the influence power of an agent $\gamma$ (represented in Table 1) while satisfying the budget constraints. 
The budget of $[0.7,0.7,0.7,0.7,0.7,0.5]$ is allocated to the agents $[8,3,10,9,5$, 4] and the resultant cost $J\left(u^{*}\right)=0.7331$. To better understand the advantage

\begin{tabular}{||ccccccccccc||}
\hline Agents & 1 & 2 & 3 & 4 & 5 & 6 & 7 & 8 & 9 & 10 \\
\hline \hline c & 1 & 1 & 1 & 1 & -1 & 1 & -1 & 1 & 1 & -1 \\
\hline Initial Opinion & 0.8 & 0.7 & 0.5 & 0.3 & -0.3 & 0 & -0.4 & 0.2 & -0.5 & 0.6 \\
\hline Centrality & 0.075 & 0.054 & 0.224 & 0.095 & -0.111 & 0.052 & -0.108 & 0.160 & 0.057 & -0.064 \\
\hline Gamma $(\gamma)$ & 0.015 & 0.016 & 0.112 & 0.067 & 0.078 & 0.052 & 0.065 & 0.128 & 0.085 & 0.101 \\
\hline
\end{tabular}

Table 1: Data

of the designed marketing strategy (strategy 1), the results obtained are compared with the ones obtained from strategy 2 and 3 . Using strategy 2, the total available budget $B=4$ is allocated uniformly among $N$ agents and the resultant cost is 0.7728 . Next, with strategy 3 , the positive budget is uniformly allocated to all the agents and the cost obtained is 0.8646 .

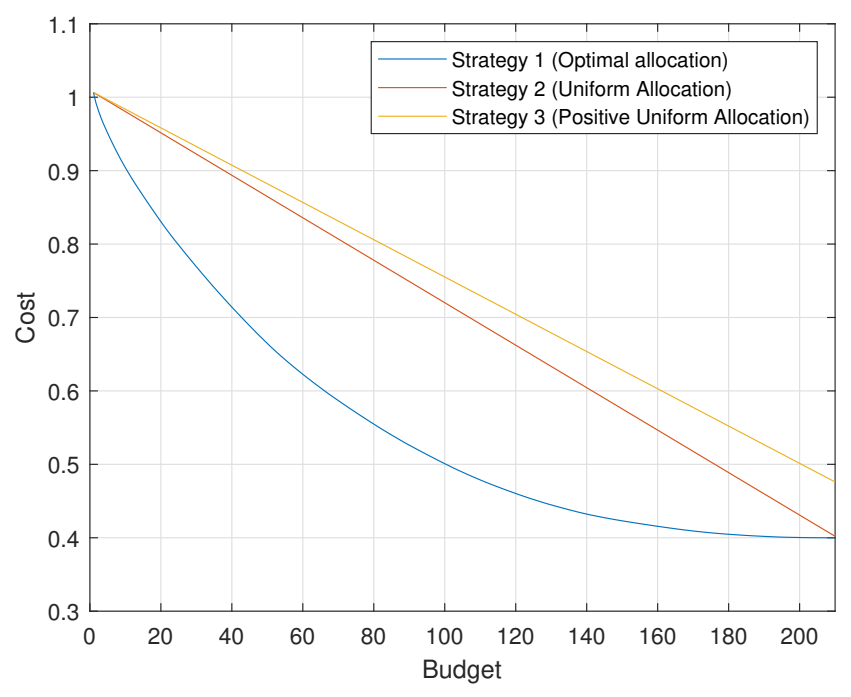

Fig. 3: Cost vs Budget.

Finally, we consider a large-scale network with 300 nodes, formed by 279 conformists and 21 contrarians. The graph is constructed in the following manner, among the 300 agents, 25 nodes are taken to be central and influence 30 to 60 agents, while the remaining 275 have 1 to 30 agents they influence. The initial opinion of the agents are generated by the formula $x_{i}(0)=-1+\frac{2 i}{N}$.

As seen in Figure 3, the cost with no control (or when budget $B=0$ ) is the same for all strategies i.e., $J(0)=1$. It is clear from the plot that the cost 
is smaller using Strategy 1 (based on Theorem 1) for any budget between 0 and $N \bar{u}=210$ compared to the other strategies. Note that when $B=N \bar{u}$, there is enough budget to allocate the maximum control to all agents, making Strategy 1 and 2 equivalent. However, for strategy 3, due to positive budget allocation even to contrarians, the cost is significantly higher $(J=0.48)$ even when $B=N \bar{u}=210$.

\section{Conclusion}

In this work, we have shown how an optimal campaign strategy can be designed for the control of opinion dynamics over a social network. The main novelty of this work, with respect to previous works on control of opinion dynamics is that we consider the presence of contrarians in the network, which have an antagonistic relationship with the other agents. The external entity or the marketer wants to bring the average opinion of all agents as close as possible to a desired opinion $d$. Interestingly, we see that the optimal strategy involves bringing the conformists closer to $d$ and the contrarians closer to $-d$, and allocating the available budget among the best agents sorted according to a centrality measure and the distance of their initial opinions to $d$ or $-d$. The future directions to this work would involve considering multiple-campaigns or a continuous control as well as the presence of a competing marketer, resulting in a non-cooperative game.

\section{A Appendix}

Lemma 1. Given an optimization problem (OP) under the following standard form

$$
\begin{aligned}
\underset{y \in \mathbb{R}^{N}}{\operatorname{minimize}} & C\left(y^{+}, y^{-}\right) \\
\text {subject to } & y_{i}^{s}-\bar{y} \leq 0, \forall i \in\{1, \ldots, N\}, s \in\{+,-\} \\
& -y_{i}^{s} \leq 0, \forall i \in\{1, \ldots, N\}, s \in\{+,-\} \\
& -B+\sum_{i=1}^{N} y_{i}^{+}+y_{i}^{-} \leq 0
\end{aligned}
$$

where $N \in \mathbb{N}, N \geq 1, \bar{y}<1, B \geq 0$ and $C(y)$ is a decreasing convex function in $y_{i}$ such that the following condition holds.

For all $i \in\{1, \ldots, N\}, \exists g(y) \geq 0$ such that

$$
\frac{\partial C(y)}{\partial y_{i}^{+}}=-k_{i} g(y) \text { and } \frac{\partial C(y)}{\partial y_{i}^{-}}=k_{i} g(y)
$$

for some $k_{i} \in \mathbb{R}$. 
Then an optimal solution $y^{*}$ to this $O P$ is given by water-filling as follows

$$
y_{\mathrm{o}(i)}^{+*}= \begin{cases}\bar{y} & \text { if } \quad i \leq\left\lfloor\frac{B}{\bar{y}}\right\rfloor \text { and } k_{\mathrm{o}(i)}>0 \\ B-\bar{y}\left\lfloor\frac{B}{\bar{y}}\right\rfloor & \text { if } \quad i=\left\lfloor\frac{B}{\bar{y}}\right\rfloor+1 \text { and } k_{\mathrm{O}(i)}>0 \\ 0 & \text { otherwise }\end{cases}
$$

and

$$
y_{\mathrm{o}(i)}^{-*}=\left\{\begin{array}{lll}
\bar{y} & \text { if } & i \leq\left\lfloor\frac{B}{\bar{y}}\right\rfloor \text { and } k_{\mathrm{o}(i)}<0 \\
B-\bar{y}\left\lfloor\frac{B}{\bar{y}}\right\rfloor & \text { if } & i=\left\lfloor\frac{B}{\bar{y}}\right\rfloor+1 \text { and } k_{\mathrm{O}(i)}<0 \\
0 & \text { otherwise }
\end{array}\right.
$$

where $0:\{1, \ldots, N\} \mapsto\{1, \ldots, N\}$ represents an ordering function which can be any bijection for Case 2 and, one satisfying $\left|k_{\mathrm{o}(1)}\right| \geq\left|k_{\mathrm{o}(2)}\right| \geq \cdots \geq\left|k_{\mathrm{o}(N)}\right|$ for Case 1.

Proof: Note that all the constraint functions of the considered OP are affine, which corresponds to sufficient conditions for applying KKT conditions. Since the OP is convex, KKT conditions are necessary and sufficient for optimality. By denoting the Lagrangian by

$$
\begin{aligned}
\ell\left(y, \lambda^{+}, \bar{\lambda}^{+}, \widehat{\lambda}, \lambda^{-}, \bar{\lambda}^{-}\right) & =C(y)+\left(\sum_{i=1}^{N} \sum_{s \in\{+,-\}} \bar{\lambda}_{i}^{s}\left(y_{i}^{s}-\bar{y}\right)-\lambda_{i}^{s} y_{i}^{s}\right) \\
& +\widehat{\lambda}^{+}\left(-B+\sum_{i=1}^{N} y_{i}^{+}+y_{i}^{-}\right) .
\end{aligned}
$$

Let us assume that $\frac{\partial C(y)}{\partial y_{i}}=k_{i} g(y)$, in this case the first necessary and sufficient condition for optimality can be simplified to write

$$
k_{i} g\left(y^{\star}\right)=\lambda_{i}^{+\star}-\bar{\lambda}_{i}^{+\star}+\widehat{\lambda}^{\star}+\lambda_{i}^{-\star}-\bar{\lambda}_{i}^{-\star}
$$

which must hold for all $i \in\{1, \ldots, N\}$. The primal feasibility conditions write

$$
0 \leq y_{i}^{s \star} \leq \bar{y} \forall i \in\{1, \ldots, N\}, s \in\{+,-\},
$$

and

$$
\sum_{i=1}^{N} y_{i}^{+\star}+y_{i}^{-\star} \leq B
$$

All the KKT multipliers must satisfy the dual feasibility conditions: $\lambda_{i}^{s \star} \geq 0$, $\bar{\lambda}_{i}^{s \star} \geq 0, \widehat{\lambda}^{s \star} \geq 0$ for all $i \in\{1, \ldots, N\}, s \in\{+,-\}$. At last, the complementary slackness conditions are given by

$$
\begin{aligned}
& \bar{\lambda}_{i}^{s \star}\left(y_{i}^{s \star}-\bar{y}\right)=0, \\
& \lambda_{i}^{s \star} y_{i}^{\star}=0, \\
& \hat{\lambda}^{\star}\left[\left(\sum_{i=1}^{N} y_{i}^{+\star}+y_{i}^{-\star}\right)-B\right]=0 .
\end{aligned}
$$


Since $g\left(y^{\star}\right)$ is identical for all $i \in\{1, \ldots, N\}$ and $s \in\{+,-\}$ and is nonnegative, we must have $\lambda_{i}^{s \star}, \bar{\lambda}_{i}^{s \star}$ and $\widehat{\lambda^{*}}$ chosen so that (18) holds. We get $y_{i}^{+} y_{i}^{-}=$ $0, \forall i \in\{1, \ldots, N\}$.

Take $y^{\star}$ from (16). Set $\lambda_{j}^{s \star}=\bar{\lambda}_{j}^{s \star}=0$ for $j=\left(\mathcal{O}\left\lceil\frac{\beta_{k}}{\bar{u}}\right\rceil\right)$ and $s=+$ if $k_{j}>0$ and $s=-$ if $k_{j}<0$ as it is the only component with a non-saturated solution. For any $n, y_{n}^{+*}=0$ if $k_{n}<0$ and $y_{n}^{-*}=0$ if $k_{n}>0$ can be imposed as the left hand side of (18) will be negative and positive correspondingly and letting $\lambda_{n}^{s} \neq 0$ will be possible.

For any $i$ such that $\mathcal{O}(i)<j$, we have $k_{i} \geq k_{j}$ and this can be satisfied by setting $y_{i}^{\operatorname{sign}\left(k_{i}\right) \star}=\bar{y}$ and having $\bar{\lambda}_{i}^{\operatorname{sign}\left(k_{i}\right) \star}>0$ and $\lambda_{i}^{s}=0$. On the other hand, for any $i$ such that $\mathcal{O}(i)>j$, we set $y_{i}^{*}=0$ and the KKT conditions are satisfied if $\bar{\lambda}_{i}^{s}=0$ and $\lambda_{i}^{s \star}>0$. The solution from (16) can also be verified to satisfy (19) and therefore, we have it satisfying all the KKT conditions.

\section{References}

1. Altafini, C.: Consensus problems on networks with antagonistic interactions. IEEE Transactions on Automatic Control 58(4), 935-946 (2013)

2. Bonacich, P., Lloyd, P.: Eigenvector-like measures of centrality for asymmetric relations. Social Networks 23, 191-201 (2001)

3. Caponigro, M., Piccoli, B., Rossi, F., Trélat, E.: Sparse feedback stabilization of multi-agent dynamics. In: Proceedings of the 55th IEEE Conference on Decision and Control. pp. 4278-4283 (2016)

4. Chowdhury, N.R., Morărescu, I.C., Martin, S., Srikant, S.: Continuous opinions and discrete actions in social networks: a multi-agent system approach. In: Proceedings 55th IEEE Conference on Decision and Control (2016)

5. Davis, J.H.: Understanding group behavior : Consensual action by small groups, vol. 1, chap. Group decision making and quantitative judgments : A consensus model, pp. 35-60. Lawrence Erlbaum Associates (1996)

6. Deffuant, G., Neau, D., Amblard, F., Weisbuch, G.: Mixing beliefs among interacting agents. Advances in Complex Systems 3, 87-98 (2000)

7. DeGroot, M.H.: Reaching a consensus. Journal of the American Statistical Association 69(345), 118-121 (1974)

8. Dietrich, F., Martin, S., Jungers, M.: Control via leadership of opinion dynamics with state and time-dependent interactions. IEEE Transactions on Automatic Control 10.1109/TAC.2017.2742139 (2017)

9. Friedkin, N.E., Johnsen., E.C.: Social influence and opinions. Journal of Mathematical Sociology. 15, 193-206 (1990)

10. Hegselmann, R., Krause, U.: Opinion dynamics and bounded confidence models, analysis, and simulation. Journal of Artificial Societies and Social Simulation 5(3) (2002)

11. Hegselmann, R., Kurz, S., Niemann, C., Rambau, J.: Optimal opinion control : The campaign problem. Journal of Artificial Societies and Social Simulation 18(3) (2015) 
12. Hendrickx, J.M.: A lifting approach to models of opinion dynamics with antagonisms. In: IEEE Conference on Decision and Control. IEEE (2014)

13. Kerckhove, C.V., Martin, S., Gend, P., Rentfrow, P.J., Hendrickx, J.M., Blondel, V.D.: Modelling influence and opinion evolution in online collective behaviour. PLoS ONE 11(6), 1-25 (2016)

14. Morărescu, I.C., Girard, A.: Opinion dynamics with decaying confidence: Application to community detection in graphs. IEEE Transactions on Automatic Controlon Automatic Control 56(8), 1862 - 1873 (2011)

15. Morărescu, I.C., Varma, V.S., Buşoniu, L., Lasaulce, S.: Space-time budget allocation policy design for marketing over social networks. Nonlinear Analysis and Hybrid Systems (under review)

16. Ohtsubo, Y., Masuchi, A., Nakanishi, D.: Majority influence process in group judgment : Test of the social judgment scheme model in a group polarization context. Group processes \& intergroup relations 5(3), 249-261 (2002)

17. Proskurnikov, A.V., Matveev, A.S., Cao, M.: Opinion dynamics in social networks with hostile camps: Consensus vs. polarization. IEEE Transactions on Automatic Control 61(6), 1524-1536 (2015) 